\title{
The scalar and electromagnetic form factors of the nucleon in dispersively improved Chiral EFT
}

\author{
Jose Manuel Alarcón* \\ Theory Center, Jefferson Lab, Newport News, VA 23606, USA \\ E-mail: alarconejlab.org
}

\begin{abstract}
We present a method for calculating the nucleon form factors of $G$-parity-even operators. This method combines chiral effective field theory ( $\chi$ EFT) and dispersion theory. Through unitarity we factorize the imaginary part of the form factors into a perturbative part, calculable with $\chi$ EFT, and a non-perturbative part, obtained through other methods. We consider the scalar and electromagnetic (EM) form factors of the nucleon. The results show an important improvement compared to standard chiral calculations, and can be used in analysis of the low-energy properties of the nucleon.
\end{abstract}

XVII International Conference on Hadron Spectroscopy and Structure - Hadron2017 25-29 September, 2017

University of Salamanca, Salamanca, Spain

*Work supported by the U.S. Department of Energy, Office of Science, Office of Nuclear Physics under contract DE-AC05-06OR23177, and by the Spanish Ministerio de Economía y Competitividad and European FEDER funds under Contract No. FPA2016-77313-P. 


\section{Introduction}

The form factors (FFs) of the nucleon parametrize the transition matrix elements of local operators between nucleon states. They provide information about the internal structure and can be related to the spatial distribution of the properties encoded in the corresponding operator. A deeper knowledge of these quantities is needed in order to understand the properties of the nucleon in terms of its QCD constituents, which is required in many experimental programs. The scalar form factor encodes the response of the nulceon under scalar probes, and plays an important role in studies of direct Dark Matter detection [1]. The electromagnetic form factors, on the other hand, provide information about the internal charge distribution of the nucleon, and are key ingredients in order to understand, and ultimately solve, the so-called "Proton Radius Puzzle" [2, 3]. Form factors of higher spin operators, like the traceless part of the QCD energy-momentum tensor, are related to the momentum and angular momentum of quarks and gluons in the nucleon, and to the forces acting on them.

In this contribution we consider the extraction of the scalar and electromagnetic form factors of the nucleon using a recently developed method called Dispersively Improved Chiral Effective Field Theory (DI $\chi$ EFT). This approach combines Chiral Effective Field Theory $(\chi$ EFT) and techniques from dispersion theory. The former has experienced important developments in the single-baryon $[4,5,6,7,8]$ and multi-baryon interactions $[9,10]$, and it has shown that it can provide important results in areas of great interest, like in searches of physics beyond the Standard Model [11, 12, 13]. A more detailed discussion about the benefits of this approach and recent results were presented in Refs. $[14,15,16]$. Other matrix elements of $G$-parity-even local operators can be studied with this approach as well, and will be considered in future publications.

\section{Formalism}

The matrix elements related to the scalar and electromagnetic FFs are

$$
\begin{aligned}
& \left\langle N\left(p^{\prime}, s^{\prime}\right)\left|O_{\sigma}(0)\right| N(p, s)\right\rangle=\sigma(t) \bar{u}\left(p^{\prime}, s^{\prime}\right) u(p, s) \\
& \left\langle N\left(p^{\prime}, s^{\prime}\right)\left|J_{\mu}(0)\right| N(p, s)\right\rangle=\bar{u}\left(p^{\prime}, s^{\prime}\right)\left[\gamma_{\mu} F_{1}(t)+\frac{i \sigma_{\mu \nu} q^{v}}{2 m_{N}} F_{2}(t)\right] u\left(p^{\prime}, s^{\prime}\right)
\end{aligned}
$$

where $O_{\sigma}(x) \equiv \hat{m} \sum_{q=u, d} \bar{q}(x) q(x)$ and $J_{\mu}(x) \equiv \sum_{q=u, d} e_{q} \bar{q}(x) \gamma_{\mu} q(x)$, being $e_{q}$ the electric charge of the quark $q, \hat{m}=\left(m_{u}+m_{d}\right) / 2, t=\left(p^{\prime}-p\right)^{2}, m_{N}$ the nucleon mass and $\sigma(t)\left(F_{1,2}(t)\right)$ the scalar (Dirac/Pauli) FFs. $F_{1}$ and $F_{2}$ can be related to the Sachs form factors in the following way

$$
G_{E}(t)=F_{1}(t)+\frac{t}{4 m_{N}^{2}} F_{2}(t) \quad G_{M}(t)=F_{1}(t)+F_{2}(t) \quad G_{E, M}^{V, S} \equiv \frac{1}{2}\left(G_{E, M}^{p} \mp G_{E, M}^{n}\right),
$$

where $G_{i}^{V, S}(i=E, M)$ are the isovector and isoscalar component of the FFs, respectively.

For nucleon matrix elements of $G$-parity-even operators, like the ones we are considering here, the imaginary part of the form factors are related to the pion form factors (the scalar pion FF, $\sigma_{\pi}$, for $O_{\sigma}$, and the electromagnetic pion FF, $F_{\pi}$, for $J_{\mu}$ ) and the $\pi \pi \rightarrow \bar{N} N$ amplitude projected into the channel with the quantum numbers of the operator $\left(f_{+}^{0}\right.$ for the scalar operator $O_{\sigma}$, and $f_{ \pm}^{1}$ for 
the electromagnetic operator $J_{\mu}$ ), if we consider $4 M_{\pi}^{2} \leq t \leq 16 M_{\pi}^{2}$ (being $M_{\pi}$ the pion mass). The relation is the following:

$$
\begin{aligned}
& \operatorname{Im} \sigma(t)=\frac{3 k_{c m}}{4 \sqrt{t}\left(m_{N}^{2}-t / 4\right)} \sigma_{\pi}^{*}(t) f_{+}^{0}(t)=\frac{3 k_{c m}}{4 \sqrt{t}\left(m_{N}^{2}-t / 4\right)} \frac{f_{+}^{0}(t)}{\sigma_{\pi}(t)}\left|\sigma_{\pi}(t)\right|^{2} \\
& \operatorname{Im} G_{E}^{V}(t)=\frac{k_{c m}^{3}}{m_{N} \sqrt{t}} F_{\pi}^{*}(t) f_{+}^{1}(t)=\frac{k_{c m}^{3}}{m_{N} \sqrt{t}} \frac{f_{+}^{1}(t)}{F_{\pi}(t)}\left|F_{\pi}(t)\right|^{2} \\
& \operatorname{Im} G_{M}^{V}(t)=\frac{k_{c m}^{3}}{\sqrt{2 t}} F_{\pi}^{*}(t) f_{-}^{1}(t)=\frac{k_{c m}^{3}}{\sqrt{2 t}} \frac{f_{-}^{1}(t)}{F_{\pi}(t)}\left|F_{\pi}(t)\right|^{2}
\end{aligned}
$$

As was noticed in Ref. [19], the ratios $J_{+}^{0} \equiv f_{+}^{0}(t) / F_{\pi}(t), J_{+}^{1} \equiv f_{+}^{1}(t) / F_{\pi}(t), J_{-}^{1} \equiv f_{-}^{1}(t) / F_{\pi}(t)$ have no contribution from the two-pion cut. Therefore the real functions $J_{+}^{0}$ and $J_{ \pm}^{1}$ are better suited for perturbative calculations than the spectral functions themselves, because are free from $\pi \pi$ rescattering effects. On the other hand, the pion FFs can be taken from experimental data (in the case of the electromagnetic FF), dispersion theory results or LQCD. This approach permits a factorization of the spectral function into a perturbative part $\left(J_{+}^{0}\right.$ and $\left.J_{ \pm}^{1}\right)$, related to the coupling of pions to nucleons, which is calculable with $\chi \mathrm{EFT}$, and a non-perturbative part related to the coupling of the operator to the pions, that we take from other sources. The functions $J_{+}^{0}$ and $J_{ \pm}^{1}$ receive contributions from the left-hand cut and a right-hand cut starting at the four-pion threshold. ${ }^{1}$ They are directly extracted from $\chi \mathrm{EFT}$, without the necessity of determining any free parameter (low-energy constant). Therefore, the results come out as predictions based on chiral calculations for $\pi N$ scattering.

For the calculation of $J_{+}^{0}$ and $J_{ \pm}^{1}$ we use $S U(2)_{F}-\chi$ EFT with relativistic nucleons and deltas. The relativistic formulation is important in order to have the correct analytic structure in the amplitudes. We take the $\pi N$ amplitudes of Ref. [4], and the low-energy constants (LECs) extracted from $\pi N$ scattering in Refs. [4, 20]. In order to avoid double counting in the contribution of the $t$-channel resonances such as the $\sigma$ or $\rho$, already present in $\sigma_{\pi}$ and $F_{\pi}$, we need to subtract it from the LECs determined in Refs. [4, 20]. To estimate this effect, we use the results of Ref. [21]. For more details about the method, we refer the reader to Refs. [15, 16].

\section{Results}

Once $J_{+}^{0}$ and $J_{ \pm}^{1}$ are determined, one can make predictions about the scalar and electromagnetic form factors. Since the quantity of interest for chiral EFT are precisely these functions, we compared the chiral predictions based on $\pi N$ scattering to the dispersive ones. The results are shown in Figs. 1. There, one observes how the the chiral prediction converges to the dispersive result at low momentum transfer, while the large $t$ part is well described once we add an estimated contribution of the N2LO (NLO+N2LO). See Refs.[15, 16] for details about this estimation.

Inserting the results for $J_{+}^{0}$ and $J_{ \pm}^{1}$ into Eqs. (2.4)-(2.6) and using the dispersive results for the pion FFs (see Refs.[15, 16]), we get the spectral functions shown in Figs. 2 (left panel) and 3. At leading order (LO) and next-to-leading order (NLO) the chiral prediction agrees very well with the dispersive results close to threshold $\left(\lesssim 0.2 \mathrm{GeV}^{2}\right)$. Beyond that energy, the N2LO corrections become necessary in order to agree with dispersion theory up to $t \approx 0.8 \mathrm{GeV}^{2}$.

\footnotetext{
${ }^{1}$ Dispersive calculations claim that the four-pion contribution is negligible below $\approx 1 \mathrm{GeV}^{2}$.
} 

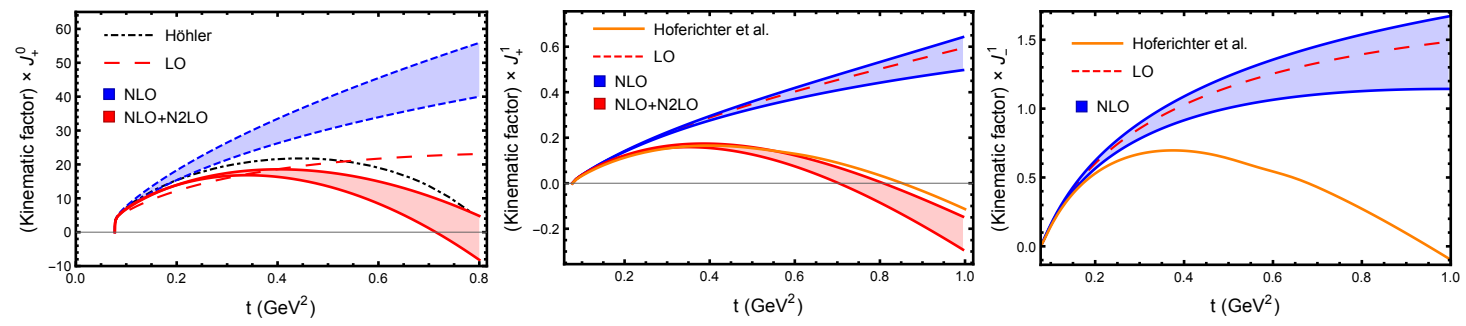

Figure 1: $\chi$ EFT results for the functions $3 k_{\mathrm{cm}} /\left(4 \widetilde{p}_{N}^{2} \sqrt{t}\right) J_{+}^{0}(t), k_{\mathrm{cm}}^{3} /\left(m_{N} \sqrt{t}\right) J_{+}^{1}(t)$ and $k_{\mathrm{cm}}^{3} /(\sqrt{2 t}) J_{-}^{1}(t)$, which enter in the last term of Eqs. (2.4), (2.5) and (2.6). Long-dashed red line: LO. Blue band: NLO. Red band: NLO + N2LO. Dashed-dotted black line: Dispersion-theoretical result of Ref. [17]. Solid orange lines: Roy-Steiner analysis results [18].

\subsection{Scalar Form Factor}

After calculating the spectral functions, one only needs to compute the dispersive integral to reconstruct the form factor. Given that part of our spectral function is calculated with $\chi \mathrm{EFT}$, we perform one subtraction in oder to suppress the high $t$ contributions in the integral. The results are shown in Fig. 2. Again, one observes that the DI $\chi$ EFT results converge well, especially below the two-pion threshold. At LO, the chiral calculation is in overall good agreement with the dispersive result. The NLO contribution helps in improving the agreement at low $t$, but is only after adding the N2LO contribution that we can reproduce very well the result from dispersion theory even up to $t \approx 0.8 \mathrm{GeV}^{2}$.
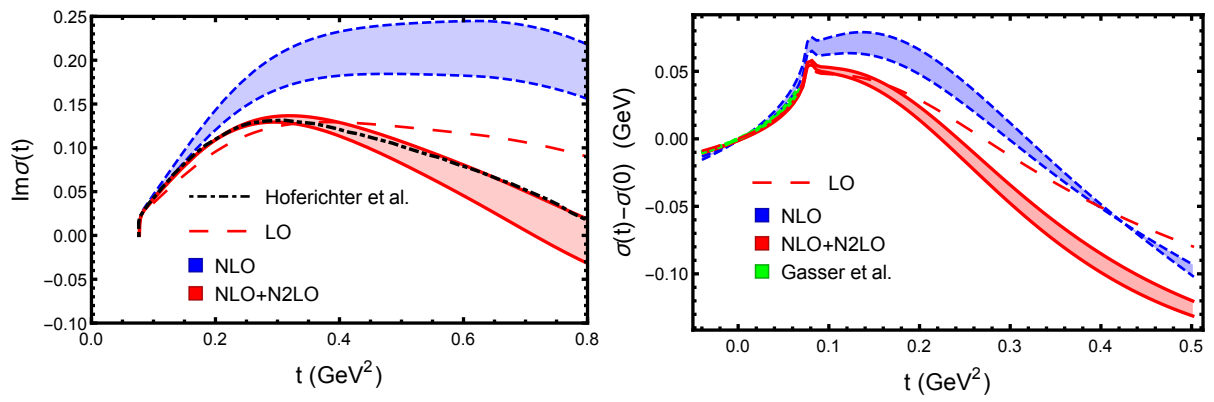

Figure 2: Nucleon scalar spectral function (left panel) and scalar FF (right panel). Red long-dashed line: LO. Blue band: NLO. Red band: NLO+N2LO. The results for the spectral function (scalar FF) are compared to the calculation of Hoferichter et al. [22] (Gasser et al [23]).

\subsection{Electromagnetic Form Factor}

With the spectral functions calculated in DI $\chi$ EFT we have access only to the isovector component of the form factor. To account for the isoscalar component, we use the results for the nucleon shown in Ref. [14]. We consider a once subtracted dispersion relation for the former, and a twice subtracted for the latter. We observe an excellent reproduction of $\operatorname{Im} G_{E}^{V}$ up to $t \approx 1 \mathrm{GeV}^{2}$, while the $\operatorname{Im} G_{M}^{V}$ would require the complete inclusion of the N2LO to achieve similar agreement (see Ref. [16]). This situation is also observed in the proton and neutron Sachs FFs. For proton and neutron the predicted $G_{E}$ agrees well with the data up to $Q^{2} \approx 0.5 \mathrm{GeV}^{2}$, while the N2LO contribution is necessary in $G_{M}$ in order to have the same agreement. 

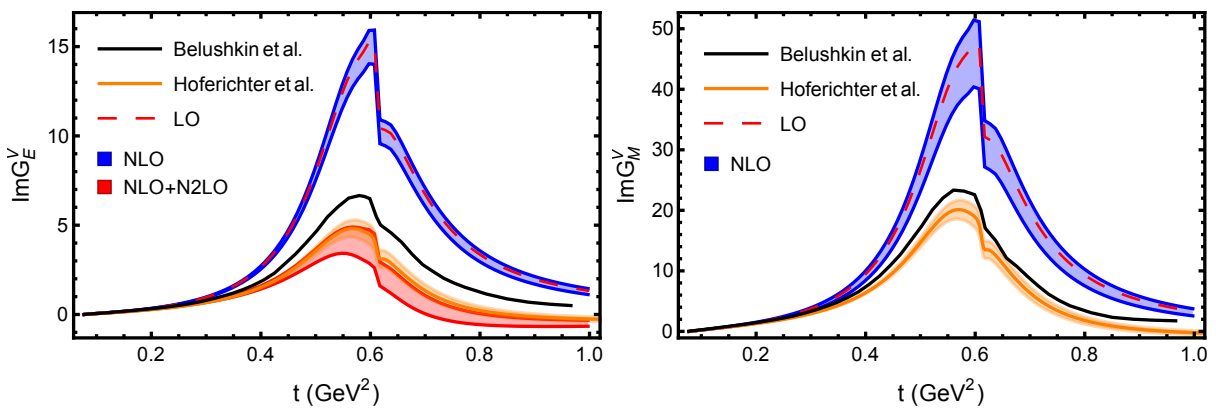

Figure 3: Nucleon isovector electromagnetic spectral functions. Long-dashed red line: LO. Blue band: NLO. Red band: NLO + N2LO. Solid back line: Result of Ref. [24]. Orange band: Roy-Steiner analysis results [18].


Figure 4: Long-dashed red line: LO. Blue band: NLO. Red band: NLO + N2LO. Black and green points correspond to experimental (Ref. [25]) and LQCD (Ref. [26]) data, respectively.

\section{Summary and Outlook}

We have presented a new method to calculate matrix elements of $G$-parity-even operators. We considered here the scalar and electromagnetic FFs of the nucleon, but it can be extended to FFs of higher spin. Through unitarity, one can factorize the spectral function into a perturbative part, calculable in $\chi \mathrm{EFT}\left(J_{+}^{0}\right.$ and $\left.J_{ \pm}^{1}\right)$, and a non-perturbative part $\left(\sigma_{\pi}\right.$ and $\left.F_{\pi}\right)$. The chiral part of the calculation shows good convergence at low momentum transfers based on the chiral results for $\pi N$ scattering up to NLO. At higher energies one needs to include the higher order contributions. An estimation of these contributions anticipates a good agreement with dispersion theory results up to $t \approx(0.8-1.0) \mathrm{GeV}^{2}$. This translates into a good agreement at the spectral function level up 
to that energies. From these spectral functions we could predict the $t$-dependence of the scalar and electromagnetic isovector FFs up to energies of $|t| \approx 0.5 \mathrm{GeV}^{2}$. We are currently working on the extension of this method to higher spin FFs and to $G$-parity-odd operators. Together with the results shown here, they will provide information related to the structure of the nucleon required in low-energy nuclear physics experimental programs and searches of new physics.

\section{References}

[1] F. Bishara, J. Brod, B. Grinstein and J. Zupan, JCAP 1702, no. 02, 009 (2017).

[2] D. W. Higinbotham, A. A. Kabir, V. Lin, D. Meekins, B. Norum and B. Sawatzky, Phys. Rev. C 93, no. 5, 055207 (2016).

[3] M. Horbatsch, E. A. Hessels and A. Pineda, Phys. Rev. C 95, no. 3, 035203 (2017).

[4] J. M. Alarcon, J. Martin Camalich and J. A. Oller, Annals Phys. 336, 413 (2013).

[5] V. Lensky, J. M. Alarcón and V. Pascalutsa, Phys. Rev. C 90, no. 5, 055202 (2014).

[6] A. N. Hiller Blin, T. Ledwig and M. J. Vicente Vacas, Phys. Lett. B 747, 217 (2015).

[7] A. N. Hiller Blin, T. Ledwig and M. J. Vicente Vacas, Phys. Rev. D 93, no. 9, 094018 (2016).

[8] A. N. Hiller Blin, Phys. Rev. D 96, 093008 (2017).

[9] S. Elhatisari et al., Phys. Rev. Lett. 117, no. 13, 132501 (2016).

[10] J. M. Alarcón et al., Eur. Phys. J. A 53, no. 5, 83 (2017).

[11] J. M. Alarcon, J. Martin Camalich and J. A. Oller, Phys. Rev. D 85, 051503 (2012).

[12] J. M. Alarcon, L. S. Geng, J. Martin Camalich and J. A. Oller, Phys. Lett. B 730, 342 (2014).

[13] J. M. Alarcon, V. Lensky and V. Pascalutsa, Eur. Phys. J. C 74, no. 4, 2852 (2014).

[14] J. M. Alarcón, A. N. Hiller Blin, M. J. Vicente Vacas and C. Weiss, Nucl. Phys. A 964, 18 (2017).

[15] J. M. Alarcón and C. Weiss, Phys. Rev. C 96, 055206 (2017).

[16] J. M. Alarcón and C. Weiss, arXiv:1710.06430 [hep-ph].

[17] G. Höhler, in Pion Nucleon Scattering. Part 2: Methods and Results of Phenomenological Analyses Landolt-Börnstein, 9b2, ed. H. Schopper (Springer, Berlin, 1983).

[18] M. Hoferichter, B. Kubis, J. Ruiz de Elvira, H.-W. Hammer and U.-G. Meissner, Eur. Phys. J. A 52, no. 11, 331 (2016).

[19] W. R. Frazer and J. R. Fulco, Phys. Rev. 117, 1609 (1960).

[20] D. Siemens, J. Ruiz de Elvira, E. Epelbaum, M. Hoferichter, H. Krebs, B. Kubis and U.-G. Meißner, Phys. Lett. B 770, 27 (2017).

[21] V. Bernard, N. Kaiser and U. G. Meissner, Nucl. Phys. A 615, 483 (1997).

[22] M. Hoferichter, C. Ditsche, B. Kubis and U. G. Meissner, JHEP 1206, 063 (2012).

[23] J. Gasser, H. Leutwyler and M. E. Sainio, Phys. Lett. B 253, 260 (1991).

[24] M. A. Belushkin, H.-W. Hammer and U. G. Meissner, Phys. Lett. B 633, 507 (2006).

[25] J. C. Bernauer et al. [A1 Collaboration], Phys. Rev. Lett. 105, 242001 (2010).

[26] C. Alexandrou et al., Phys. Rev. D 96, no. 3, 034503 (2017). 